SpeechMagic 7 jetzt auch in Citrix-Umgebungen

Die Spracherkennungssoftware Nuance SpeechMagic 7 ist jetzt „CitrixReady“zertifiziert. Das berichtet der Hersteller Nuance Communications. Damit wird der reibungslose Einsatz in Citrix-Umgebungen von Kliniken, Großpraxen und medizinischen Einrichtungen bestätigt. Die Zeit zwischen Diktat und den auf dem Bildschirm erscheinenden Wörten ist kaum mehr wahrnehmbar, so Nuance. SpeechMagic 7 unterstützt Mikrofone von Philips und ab Release 2 auch von Grundig Business Systems.

www.nuance.de

\section{Netzübergreifendes Schutzkonzept gesucht}

Das Smartphone erfreut sich zunehmender Beliebtheit, nicht zuletzt dank der offenen Betriebssysteme. Doch damit sind auch besondere Sicherheitsrisiken verbunden. Das Bundesministerium für Bildung und Forschung fördert deshalb mit rund 2,8 Millionen Euro das Forschungsprojekt ASMONIA (Attack analysis and Security concepts for MObile Network infrastructures, supported by collaborative Information exchAnge.) Ziel des Projekts ist es, ein netzübergreifendes Schutzkonzept für Mobilfunknetze zu entwickeln, u.a. ein netzübergreifendes Frühwarnsystem für Mobilfunknetze der 4. Generation.

www.bmbf.de

www.asmonia.de

\section{Google-Webbrowser Chrome in Version 7}

Der Suchmaschinenbetreiber Google bietet jetzt eine stabile Version 7 des Chrome-Browsers zum Download an. Nach Google-Angaben wurden Hunderte Fehler behoben, die in der Version 6 des Browsers enthalten waren. Außerdem wurde der HTML5-Parser implementiert, der für mehr Kompatibilität zu anderen Browsern sorgt. Google bietet zudem ein Dialogfenster an, das bei der Verwaltung geblockter Cookies hilft. Und Mac OS XNutzer freuen sich über vollen Applescript-Support. Mittels File API lassen sich nun ganze Verzeichnisse hochladen. eb

www.google.com/chrome/

\title{
Sicherheitsrisiko KV SafeNet - was ist dran?
}

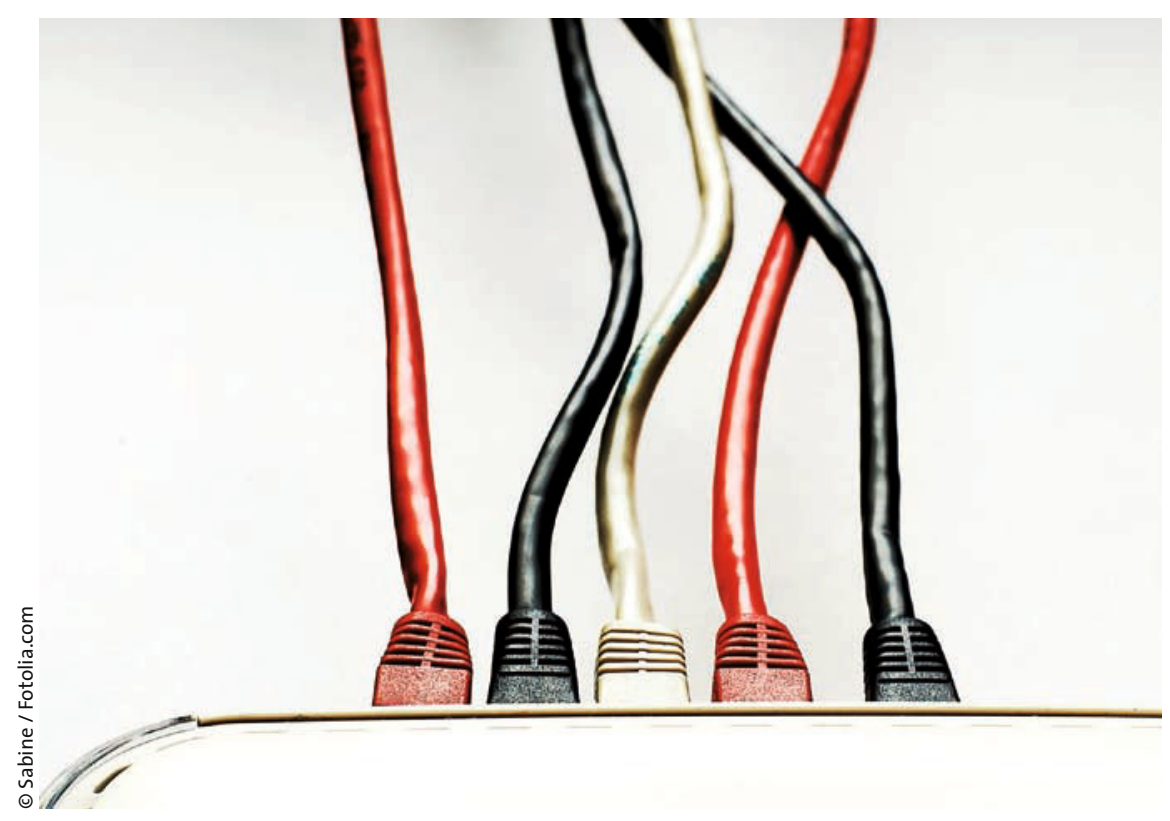

Für die Online-Abrechnung brauchen Praxen eine Anbindung an die KV-Server, das KV SafeNet soll eine sichere Variante sein.

\section{Das KV SafeNet ist wegen angeblicher Sicherheitslücken in die Kritik geraten. Doch wie funktioniert es überhaupt, und wo könnten Risiken lauern?}

ie Pflicht zur Online-Abrechnung steht vor der Tür - ab 2011 soll sie für Vertragsärzte gelten -, und ausgerechnet jetzt ist das KV SafeNet wegen angeblicher Sicherheitsrisiken unter Beschuss geraten. Dabei galten gerade das SafeNet und die diversen Abwandlungen einzelner KVen als besonders sichere Anbindungen an die KV-Rechner. Doch was ist dran an den vermeintlichen Sicherheitslücken?

Dazu muss man zunächst den Vorwurf kennen: Angeblich soll es beim KV SafeNet-Router mehrere offene Ports (Schnittstellen) geben. Diese will zumindest der Informatiker Lew Palm, der eine psychotherapeutische Praxis betreut, ausfindig gemacht haben. Er erhebt in einem mehrseitigen Papier, das der „Ärzte Zeitung "vorliegt, aber ebenso den Vorwurf, dass nicht offengelegt würde, mit welcher Verschlüsselung der Router arbeite.

Viele technische Begriffe, die sich einfach erklären lassen. Zunächst einmal ist das SafeNet keine offene Verbindung ins Internet, sondern ein sogenanntes virtuelles privates Netzwerk (VPN) - also eine Art Intranet, zu dem nur Personen mit vorher angelegten Berechtigungen $\mathrm{Zu}$ griff haben. Das VPN schottet nach Angaben der KBV die Verbindung vom Internet $\mathrm{ab}$ und gewährleistet einen sicheren Datenaustausch mit dem Rechenzentrum der jeweiligen $\mathrm{KV}$.

\section{Nur über die Blackbox kommen Praxen ins Netz}

Um in das Netz zu kommen, brauchen Praxen aber auch die Blackbox beziehungsweise den KV SafeNet-Router. 